\title{
$\sigma_{\text {and }}$ \\ CHEST Non-adherence in difficult asthma: time to take CLINIC it seriously
}

\author{
Liam G Heaney, ${ }^{1}$ Rob Horne ${ }^{2}$
}

\begin{abstract}
${ }^{1}$ Centre for Infection and immunity, Health Sciences Building, Queens University Belfast, Belfast, UK ${ }^{2}$ Centre for Behavioural Medicine, The School of Pharmacy, University of London, UK
\end{abstract}

\section{Correspondence to}

Dr Liam Heaney, Centre for Infection and Immunity, Queen's University of Belfast, Level 8, Belfast City Hospital, Lisburn Road, Belfast BT9 7AB, UK; heaney@qub.ac.uk

Received 27 March 2011 Accepted 20 May 2011 Published Online First 18 June 2011

\begin{abstract}
Recent studies have demonstrated a high prevalence of non-adherence with anti-inflammatory medication in patients referred for specialist assessment with difficultto-control asthma. As well as poor asthma outcome and increased healthcare cost, failure to detect nonadherence makes identification of true treatmentresistant/refractory asthma challenging. This is because guideline definitions of refractory asthma are all predicated on failure to respond to high-dose antiinflammatory therapy but do not state how adherence with this therapy should be assessed. With the advent of novel expensive biological therapies, the systematic identification of non-adherence becomes more essential to avoid targeting therapies at an inappropriate patient group. Novel biomarkers of steroid exposure, in combination with more traditional surrogate measures such as prescription filling assessment, may allow more objective assessments of non-adherence to be developed in the future. When identified, non-adherence can potentially be targeted and improved, but the key challenge is to empower patients to make informed choices about medicines rather than decisions influenced by misplaced beliefs about benefit and harm. There is an urgent need for the systematic development of individualised interventions which allow non-adherence to be effectively managed. Thus, non-adherence must become a priority in the clinical assessment of difficultto-control asthma because addressing non-adherence is likely to deliver greater benefits in this group than any novel treatment. It is essential that future research examines strategies and interventions to address nonadherence in subjects with difficult-to-control asthma.
\end{abstract}

Asthma is a leading preventable cause of morbidity, mortality and cost, estimated to affect 300 million people worldwide. ${ }^{1} 2$ Despite being prescribed treatment at Global Initiative for Asthma (GINA) steps 4 and 5 , approximately $5-10 \%$ of adult patients remain difficult to control with persisting symptoms and frequent exacerbations. ${ }^{3} 4$

The term 'compliance' has mostly been superseded by the term 'adherence', which recognises a patient's right to choose to take medication or engage with other healthcare-related advice and to remove the concept of blame if this does not occur. 'Concordance' includes the concept of healthcare provider/patient agreement on therapeutic decisions that incorporate their respective views, and acknowledges that there may be (potentially) opposing views. ${ }^{5}$

Adherence is low in many chronic conditions including asthma of different severities. ${ }^{6}$ Recent
UK studies have specifically looked at this in patients with difficult-to-treat asthma referred to specialist centres for assessment. In a Belfast study, 63 of 182 patients (35\%) had filled $<50 \%$ of prescriptions for inhaled combination therapy and 57 (88\%) admitted low adherence after initial denial. ${ }^{7}$ Low adherence was more common in women and was associated with recurrent hospital admission and use of nebulised bronchodilators. In a study in Leicester, 75 of 115 patients $(65.2 \%)$ on inhaled corticosteroids and 65 of 108 (60.1\%) on inhaled long-acting $\beta_{2}$ agonists had $<80 \%$ prescription filling (in the Belfast study $<80 \%$ prescription filling was $64 \%) .^{8}$ Patients with low adherence had lower mean (SD) post-bronchodilator forced expiratory volume in $1 \mathrm{~s}$ (75.4 (20.9) vs 84.3 (23.5), $\mathrm{p}<0.05)$, were more likely to have been ventilated for asthma (19.2\% vs $2.6 \%, p=0.02)$ and had higher sputum eosinophils (geometric mean (log SD) $4.6(0.66) \%$ vs $2.3(0.54) \%, p=0.05)$. In a paediatric study from London, prescription filling was assessed as part of a home visit ${ }^{9}$ with $30 \%$ of children filling prescriptions for $<50 \%$ of inhaled maintenance therapy; medication issues encompassing adherence, an unsuitable device or poor technique were felt to contribute to poor control in 34 children (48\%).

The situation is similar for adherence to oral steroids. In the Belfast study 25 of 51 patients (49\%) were found to be non-adherent to prednisolone using a cortisol/prednisolone assay; again, patients only admitted low adherence when confronted with objective measurements. ${ }^{7}$ In another adult study involving a London hospital inpatient assessment, 9 of 28 subjects (32\%) prescribed $\geq 15 \mathrm{mg}$ prednisolone per day had either non-detectable prednisolone or non-suppression of cortisol. ${ }^{10}$ In the Leicester study, using prescription records alone, 13 of 50 (26\%) had low adherence with oral prednisolone. ${ }^{8}$ It is important to note that many of these non-adherent patients with difficult-to-control asthma were referred from other specialist physicians $37 \%$ in the Belfast study, $100 \%$ in London paediatric and adult studies), suggesting that this problem is not routinely identified in specialist care. These studies would support prednisolone assays, like theophylline assays, becoming routinely available in dedicated Difficult Asthma Services to allow objective assessment of adherence with these medications.

What are the implications of not identifying nonadherence in this population? The first and most obvious problem is poor asthma outcome, particularly in terms of increased unscheduled healthcare 
use and increased near fatal events. ${ }^{7-9}$ A further consequence is increased healthcare cost and, based on the Belfast data and assuming $5 \%$ of the 5.1 million UK patients with asthma have difficult-to-control asthma, reduction in hospital admission alone (including offsetting increased drug costs) could deliver a potential annual saving to the NHS of about $£ 43$ million. ${ }^{11}$

The next consequence of unidentified low adherence relates to our ability to identify and thus investigate treatment-resistant or refractory asthma. Most definitions of treatment-resistant/ refractory asthma are based on failure to respond to high-dose asthma therapy and thus predicated on adherence with this treatment, ${ }^{3}{ }^{4}$ but do not state how this should be assessed or addressed. The WHO statement on severe asthma acknowledged this problem and defined asthma severity on the basis of current clinical control, listing three situations where this could occur: untreated severe asthma (eg, treatment not available for economic reasons), difficult-to-treat severe asthma (the clinical problem where non-adherence, comorbidities and other reasons may be the mechanism for persistent symptoms) and treatmentresistant severe asthma (where adherence has been demonstrated hence 'resistance' to currently available therapies). ${ }^{12}$ One consequence of an inappropriate diagnosis of 'refractory' or 'treatment-resistant' asthma is that newer expensive therapies will be given to the wrong patient group. For example, the Health Technology Appraisal for omalizumab by the National Institute for Health and Clinical Excellence (NICE) stated that it should be given to subjects with severe persistent allergic asthma as an 'add-on therapy to optimised standard therapy', with the latter described as 'a full trial of, and documented compliance with, inhaled high-dose corticosteroids and longacting $\beta_{2}$ agonists in addition to leukotriene receptor antagonists, theophyllines, oral corticosteroids and $\beta_{2}$ agonist tablets and smoking cessation where clinically appropriate'. ${ }^{13}$ Again, however, it is not stated how adherence should be assessed or identified. In addition, to make Omalizumab economically cost effective, NICE targeted the group of patients who were 'expensive', specifically patients with two or more hospital admissions for asthma or one hospital admission plus two severe exacerbations requiring treatment/monitoring in an $\mathrm{A} \& \mathrm{E}$ unit. Based on the Belfast study where recurrent admission was more common with non-adherence, this stipulation potentially targets a non-adherent population. The advent of novel expensive anti-eosinophilic therapies such as mepolizumab could again theoretically target persistent airways eosinophilia in patients who are non-adherent with inhaled steroid therapy.

If non-adherence is identified in difficult-to-control asthma, can anything be done about it? A recent Cochrane review of adherence interventions examined data in 13 asthma intervention studies. ${ }^{14}$ The studies were done in different clinical settings and none would have been classified as a difficult asthma population using current definitions; the interventions were usually delivered within a management plan and some studies included mixed populations (eg, asthma and chronic obstructive pulmonary disease). Six of the studies reported adherence improvement using different measures, although some of these were based on patient self-report and physician estimate and only two by prescription records. However, the improvements were modest and short-lived, with only three studies showing a change in any healthcare outcome such as improved peak flow or reliever use. The review concluded that the evidence that we can modify adherence is '... surprisingly weak...', but it acknowledged that 'increasing effectiveness of adherence interventions was likely to have a greater impact than improvement in specific medical treatments'. Recent explanatory research has advanced our understanding of non-adherence in chronic conditions. ${ }^{15}$ It is a common problem representing a failure of the healthcare system, and is best understood as a variable behaviour with intentional and unintentional causes-unintentional non-adherence is linked to limitations in capacity or resources whereas intentional non-adherence is the product of a decision informed by beliefs, emotions and preferences. Adherence is influenced by the patients' beliefs about medicines-in particular, how they judge their personal need for the treatment (necessity beliefs) relative to their concerns about potential adverse effects. ${ }^{16}{ }^{17}$ Non-adherence is often a hidden problem; as the experience in Belfast shows, patients are often reluctant to reveal non-adherence and the associated doubts and concerns about treatment to clinicians for fear of offending them. Many patients believe that lack of faith in the treatment will be interpreted by the clinician as a lack of faith in the prescriber, and any adherence support should be 'menu-driven'-that is, tailored to the needs of the individual. Using such an approach in a small randomised controlled pilot study, we have recently shown that, in the $35 \%$ of patients referred to our centre with low adherence as the primary cause of difficult-tocontrol asthma, identifying and confronting the problem in a medical concordance interview induced sustained behavioural change. ${ }^{18}$ In those subjects with persistent non-adherence, a more complex menu-driven behavioural intervention also demonstrated improved prescription filling and a trend to better outcome. ${ }^{18}$ Such approaches need to be studied in multicentre clinical trials because, if even moderately successful, they are likely to deliver a substantial improvement in outcome in this patient group.

What of the future? A review commissioned by the National Institute for Health Research considered why adherence interventions yield such disappointing effects ${ }^{19}$ and recommended the systematic development of individualised interventions, an approach recently endorsed by NICE. ${ }^{20}$ This will involve eliciting and addressing the perceptual barriers (eg, beliefs, preferences and emotions) affecting motivation to start and continue taking medication, as well as the practical barriers (eg, capacity and resources) influencing the ability to adhere to the treatment.

However, the first step remains the identification of nonadherence. The cheapest and easiest method is to ask patients, but self-report is notoriously unreliable and inaccurate. However, the accuracy and reliability of self-report can be improved by communicating a 'no-blame' approach that sanctions non-adherence. ${ }^{16} 17$ More objective measures of nonadherence are still required and the development of biomarkers and/or objective tests of steroid exposure would be valuable in difficult asthma where complex and expensive treatments are being considered. With regard to a potential biomarker, there are a number of genes which are rapidly and consistently upregulated after steroid exposure ${ }^{21}$ and some of these provide attractive targets to develop a marker of adherence to steroid therapy. However, it seems probable that-as with other objective measures of drug adherence such as theophylline and prednisolone serum levels or the use of smart chips-any such biomarker will have to be combined with other measures such as effective self-report methods and surrogates such as prescription filling, coupled with a no-blame approach to discussion. For clinical practice, the key challenge is to utilise these different measures to bring non-adherence out into the open by facilitating an honest and open discussion. Developing technological approaches such as better detection methods may facilitate this discussion but are unlikely to deliver better clinical outcomes without better methods for negotiating treatments with 
patients and supporting informed choice and optimal adherence. A recent EU directive listed improving adherence as one of 10 priorities to reduce the impact of asthma on individuals and society $^{22}$ but, while low adherence is described as a potential mechanism for difficult-to-control asthma in national and international asthma guidelines, precise guidance on how this should be identified and managed has so far been absent from international guidelines for managing difficult-to-control asthma. $^{23} 24$

In conclusion, non-adherence remains a significant problem in difficult-to-control asthma. There has been a failure to systematically implement processes and clinical tools which have been shown to help identify and address non-adherence. The key challenge is to empower patients to make informed choices about medicines rather than decisions influenced by misplaced beliefs about benefit and harm. There are many consequences of this failure but, given the advent of novel expensive parenteral therapies for more difficult-to-manage asthma, it has now become imperative that non-adherence is targeted and clinical trials supported to examine interventions to address the problem in subjects with difficult asthma.

Competing interests None.

Contributors Both authors conceived and wrote the article and reviewed and approved the final manuscript.

Provenance and peer review Not commissioned; externally peer reviewed.

\section{REFERENCES}

1. Masoli M, Fabian D, Holt S, et al; Global Initiative for Asthma (GINA) Program. The global burden of asthma: executive summary of the GINA Dissemination Committee report. Allergy 2004;59:469-78.

2. World Health Organization. Asthma. Geneva: World Health Organization. http:// www.who.int/topics/asthma/en/

3. Barnes PJ, Woolcock AJ. Difficult asthma. Eur Respir J 1998;12:1209-18.

4. Anon. Proceedings of the ATS workshop on refractory asthma: current understanding, recommendations, and unanswered questions. American Thoracic Society. Am J Respir Crit Care Med 2000;162:2341-51.

5. Royal Pharmaceutical Society of Great Britain. From Compliance to Concordance; Achieving Shared Goals in Medicine Taking. London: Royal Pharmaceutical Society of Great Britain and Merck Sharp \& Dohme, 1997.

6. Horne R. Compliance, adherence, and concordance: implications for asthma treatment. Chest 2006;130(Suppl 1):65S-72.

7. Gamble J, Stevenson M, McClean E, et al. The prevalence of non-adherence in difficult asthma. Am J Respir Crit Care Med 2009:180:817-22.
8. Murphy AC, Proeschel A, Linnett ME, et al. Identifying non-adherence with asthma medication and the relationship to clinical outcomes amongst adults with difficult-tocontrol asthma. European Academy of Allergy and Clinical Immunology, 2010;65 (Suppl IV):xlii.

9. Bracken $\mathbf{M}$, Fleming $L$, Hall $P$, et al. The importance of nurse-led home visits in the assessment of children with problematic asthma. Arch Dis Child 2009;94:780-4.

10. Robinson DS, Campbell DA, Durham SR, et al; Asthma and Allergy Research Group of the National Heart and Lung Institute. Systematic assessment of difficult-to-treat asthma. Eur Respir J 2003;22:478-83.

11. O'Neill C, Gamble J, Lindsay JT, et al. The impact of non-adherence to inhaled longacting $\beta 2$-agonist/steroid combination therapy on healthcare costs in difficult to treat asthma. Pharmaceut Med 2011. under review.

12. Bousquet J, Mantzouranis E, Cruz AA, et al. Uniform definition of asthma severity, control, and exacerbations: document presented for the World Health Organization consultation on severe asthma. J Allergy Clin Immunol 2010;126:926-38.

13. National Institute for Health and Clinical Excellence. Asthma (uncontrolled): omalizumab. London: National Institute for Health and Clinical Excellence. http:// guidance.nice.org.uk/TA133.

14. Haynes RB, Ackloo E, Sahota N, et al. Interventions for enhancing medication adherence. Cochrane Database Syst Rev 2008;(2):CD000011.

15. Horne R, Weinman J. Self-regulation and self-management in asthma: exploring the role of illness perceptions and treatment beliefs in explaining non-adherence to preventer medication. Psychol Health 2002;17:17-32.

16. Clatworthy J, Price D, Ryan D, et al. The value of self-report assessment of adherence, rhinitis and smoking in relation to asthma control. Prim Care Respir J 2009; 18:300-5.

17. Menckeberg TT, Bouvy ML, Bracke M, et al. Beliefs about medicines predict refill adherence to inhaled corticosteroids. J Psychosom Res 2007;64:47-54.

18. Gamble J, Stevenson M, Heaney LG. A study of a multi-level intervention to improve non-adherence in difficult to control asthma. Respir Med. 2011 Apr 19. [Epub ahead of print]. doi:10.1016/j.rmed.2011.03.019

19. Horne R, Kellar I. Chapter 6: Interventions to facilitate adherence. In: Horne R, Weinman J, Barber N, et al. eds. Concordance, Adherence and Compliance in Medicine Taking: A Conceptual Map and Research Priorities. London: National Institute for Health Research (NIHR) Service Delivery and Organisation (SDO) Programme. http://www.sdo.nihr.ac.uk/files/project/SD0_FR_08-1412-076_V01.pdf (accessed 2011)

20. National Institute for Health and Clinical Excellence (NICE). Medicines Adherence: Involving Patients in Decisions about Prescribed Medicines and Supporting Adherence (CG76): Ouick Reference Guide. http://guidance.nice.org.uk/ CG76 (accessed January 2009).

21. Woodruff PG, Modrek B, Choy DF, et al. T-helper type 2-driven inflammation defines major subphenotypes of asthma. Am J Respir Crit Care Med 2009:180:388-95.

22. Holgate S, Bisgaard $H, B j e r m e r ~ L$, et al. The Brussels declaration: the need for change in asthma management. Eur Respir J2008;32:1433-42.

23. Global Initiative for Asthma (GINA).Global Strategy for Asthma Management and Prevention. $2010 \mathrm{http}: / /$ www.ginasthma.org.

24. British Thoracic Society/Scottish Intercollegiate Guidelines Network. British guideline on the management of asthma. Thorax 2008;63(Suppl 4): iv1-121. 